Article

\title{
Fluorine-Free Superhydrophobic Coatings Based on Silicone and Functionalized Colloidal Silica
}

\author{
Hui Lei ${ }^{1, *}$, Jun Xiao ${ }^{1}$, Mingna Xiong ${ }^{1}$, Liping Zheng ${ }^{1}$ and Qixin Zhuang ${ }^{2}$ \\ 1 GE (China) Research and Development Centre, Shanghai 201203, China; jxiao27@163.com (J.X.); \\ txiong@mmm.com (M.X.); liping.zheng@sabic.com (L.Z.) \\ 2 Key Laboratory of Advanced Polymer Materials of Shanghai, School of Materials Science and Engineering, \\ East China University of Science and Technology, Shanghai 200237, China; qxzhuang@ecust.edu.cn \\ * Correspondence: leihuilh@hotmail.com
}

Received: 1 February 2019; Accepted: 28 February 2019; Published: 28 February 2019

check for updates

\begin{abstract}
Recently, fluorine materials have been a serious cause of environmental concern. In response, a novel fluorine-free superhydrophobic coating is presented in this paper. A superhydrophobic coating based on silicone and surface-modified colloidal silica is explored and exploited. First, a superhydrophobic coating, based on silicone resins and fluorine group-modified colloidal silica, is developed. Then, the fluorine group-modified colloidal silica is replaced by octyl-modified colloidal silica, a superhydrophobic coating based on fluorine-free materials, octyl-functionalized colloidal silica, and epoxy-modified silicone. The hydrophobicity and coating integrity were investigated, and the fluorine-free coating shows good superhydrophobicity and coating integrity. The result demonstrates the feasibility of a fluorine-free superhydrophobic coating, thus providing an effective solution to the environmental problems caused by fluorine chemicals.
\end{abstract}

Keywords: superhydrophobic coating; colloidal silica; silicon; fluorine-free

\section{Introduction}

The wettability of solid surfaces plays a very important role in our daily lives, as well as in many industrial processes. For example, the leaves of certain plants and many insects exhibit superhydrophobicity [1-3]. This means water does not show any affinity to their surfaces, causing the water drops to bounce and roll instead of sliding on the surface. When water droplets roll off such surfaces, they drag along dirt particles, which explains their self-cleaning property. The lotus leaf is a well-known example of a plant that has a self-cleaning surface. The secret behind it was discovered by Barthlott and his co-workers in 1997 [4,5]. Lotus leaves are covered by many pillars with micrometer-scale dimensions, which are decorated with sub-micrometer wax crystals. Consequently, the leaves possess dual-scale roughness, which promotes hydrophobicity [6]. Recently, superhydrophobic surfaces, with a contact angle larger than $150^{\circ}$ and a roll-off angle less than $5^{\circ}$, have attracted significant attention because of their self-cleaning properties and their potential for practical applications in a wide range of fields. This includes self-cleaning coatings on buildings $[7,8]$, oil-water separation [9,10], anti-icing coatings [11-14], anti-corrosion coatings [15,16], anti-wetting textiles [17,18], and drag reduction devices $[19,20]$.

Surface wettability is manipulated by two crucial factors. One is the chemical composition, and the other is the topography of the solid surface. Superhydrophobic surfaces can be generated by incorporating low surface energy materials, by creating multi-scale topography, or by combining both. For superhydrophobic coatings in a commercial application, a simple and cost-effective fabrication process and easily available raw materials are desired. However, many of the current preparation routes, such as plasma etching [21] and chemical vapor deposition [22], are not very straightforward, 
i.e., they require complex equipment, multiple manufacturing steps or are time- or cost-consuming. For most applications, the coating integrity of the superhydrophobic surface is important for maintaining long-term superhydrophobicity [23]. In the literature, many superhydrophobic surfaces were developed, but their coating integrity was usually poor, and their superhydrophobicity was easily lost. In this paper, the major target is to develop a coating with sustainable superhydrophobicity and good coating integrity simultaneously.

Currently, most superhydrophobic coatings are based on fluorine materials due to their excellent hydrophobic properties. In our previous study [24], a unique superhydrophobic coating system was developed with fluorine-functionalized colloidal silica and a cross-linkable fluorocopolymer as a filler and matrix, respectively, to yield superhydrophobicity, good mechanical stability, and strong adhesion with substrates. The effects of many factors, such as particle surface functionalities, volume fraction and dimension on hydrophobicity, and the integrity of coatings, were systematically investigated. Combining the hydrophobicity results with the coating integrity results, the optimized formulation was determined to include a $60 \%$ volume of fluorine-functionalized colloidal silica with a particle size of $340 \mathrm{~nm}$ and a fluorocopolymer. However, long-chain fluoropolymers are reported to release substances such as perfluorooctyl carboxylates or perfluorooctyl sulfonates into the environment. These substances persist in the environment, accumulate in organisms, and are suspected of being "highly toxic" [25-27]. The US Environmental Protection Agency (EPA) has enacted stricter regulations prohibiting the use of these materials. Therefore, a fluorine-free superhydrophobic coating with good mechanical properties is desired to solve this environmental problem. Based on the optimized formulation identified in our previous work, in this paper, the fluorine components are replaced by fluorine-free materials step by step. First, the fluorocopolymer is replaced by silicone materials, and thus, a superhydrophobic coating system from fluorine-functionalized colloidal silica and silicone is studied. Then, the fluorine-functionalized colloidal silica is further replaced by fluorine-free colloidal silica-a superhydrophobic system with fluorine-free materials, which consists of fluorine-free colloidal silica and silicone. Its superhydrophobicity and the coating integrity are also studied in this paper.

\section{Experiment}

\subsection{Materials}

All the solvents were purchased from Sinopharm Chemical Reagent Co., Ltd., (Shanghai, China). Tetraethyl orthosilicate (TEOS) was obtained from Wuhan University Silicone New Material Co., Ltd., (Wuhan, China). (Tridecafluoro-1,1,2,2-tetrahydrooctyl) methyldichlorosilane and n-octyldimethylchlorosilane were purchased from Gelest, Inc. (Morrisville, PA, USA). Colloidal silica with a particle size of $12 \mathrm{~nm}$ was purchased from Fuso Chemical Co., Ltd., (Kyoto, Japan). Fluorocopolymer (a random copolymer of different fluorine-functionalized units whose chemical structure is shown in Figure 1) and hexamethylene diisocyanate (HDI) were supplied by Xuzhou Zhangyan Fluorine Chemical Co., Ltd., (Xuzhou, China). Pure silicones (TSR116, TSR144, TSR145, and TSR127B), modified silicones (TSR194, TSR175, and TSR171), and curing catalyst (CR12) were provided by Momentive Advanced Materials (Waterford, NY, USA). The basic structure of pure silicone resin is shown in Figure 2. For the modified silicone resins, the basic structures are similar and some of the R groups are replaced with functional groups accordingly. TSR194 is an epoxy-modified silicone, and TSR171 is an acrylic-modified silicone. TSR 175 is a two-component urethane-modified silicone.

\subsection{Synthesis of Colloidal Silica and Preparation of Fluorine- and Octyl-Functionalized Colloidal Silica}

Colloidal silica with a particle size of $340 \mathrm{~nm}$ was synthesized by the Stober method. The detailed synthetic approach is described in our previous paper [24]. The detailed procedure for preparing fluorine-functionalized colloidal silica $(340 \mathrm{~nm})$ is also described in our previous paper. The octyl-functionalized colloidal silica was synthesized by the same procedures. 


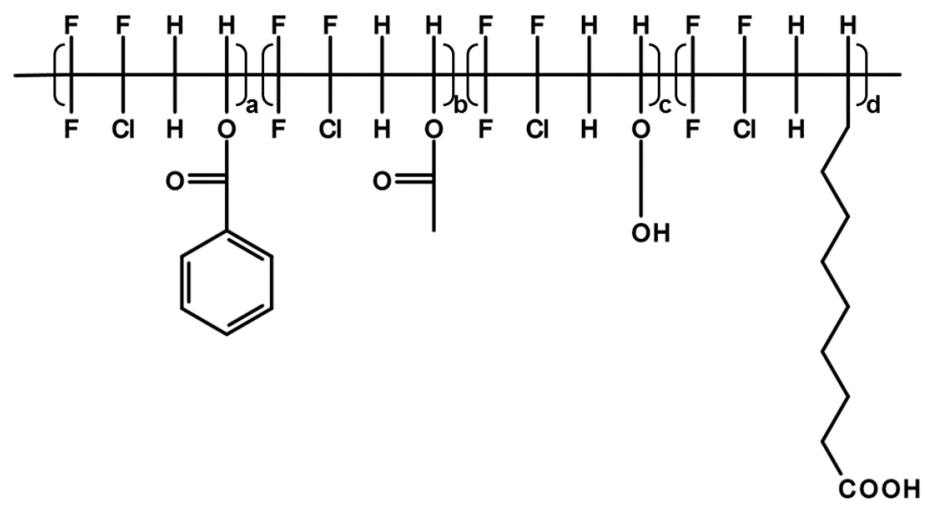

Figure 1. Chemical structure of the fluorocopolymer.<smiles>[R][Si]([R])(OC)OC</smiles>

D (Di-functional group)<smiles>[R][Si](OC)(OC)OC</smiles>

T (Tri-functional group)<smiles>[R][R]1([R])O[Si]([R])(OC)O[Si]([R])(OC)O[Si]2([R])O[Si]([R])([R])O[Si]([R])(OC)O[Si]([R])(OC)O[Si]([R])(OC)O[Si]([R])(OC)O[Si]([R])(O1)O2</smiles>

$\mathrm{R}=\mathrm{CH}_{3}$ or phenyl

Figure 2. Basic structure of the pure silicone resins.

\subsection{Preparation of Superhydrophobic Coatings}

The fabrication process of a superhydrophobic coating is shown in Figure 3. Functionalized colloidal silica was mixed with different polymers at a volume ratio of $60 \%$ by ultra-sonication for $15 \mathrm{~min}$, followed by magnetic stirring for an additional $30 \mathrm{~min}$. Designated amounts of HDI (mass ratio: fluorocopolymer/HDI $=4: 1$ ) or silicone resin curing catalyst CR12 (mass ratio: silicone resin/catalyst $=50: 1$ ) was dissolved in cyclohexanone and then added to the above mixture by magnetic stirring for about $10 \mathrm{~min}$. The coating was prepared by spraying the mixture onto clear glass slides or a stainless-steel plate (F-2 spray gun (Fuzhou Hengsheng Hardware Co.,Ltd., Fuzhou, China), air flow rate: $3 \mathrm{~kg} / \mathrm{cm}^{2}$ ), followed by curing at $100{ }^{\circ} \mathrm{C}$ for $1 \mathrm{~h}$.
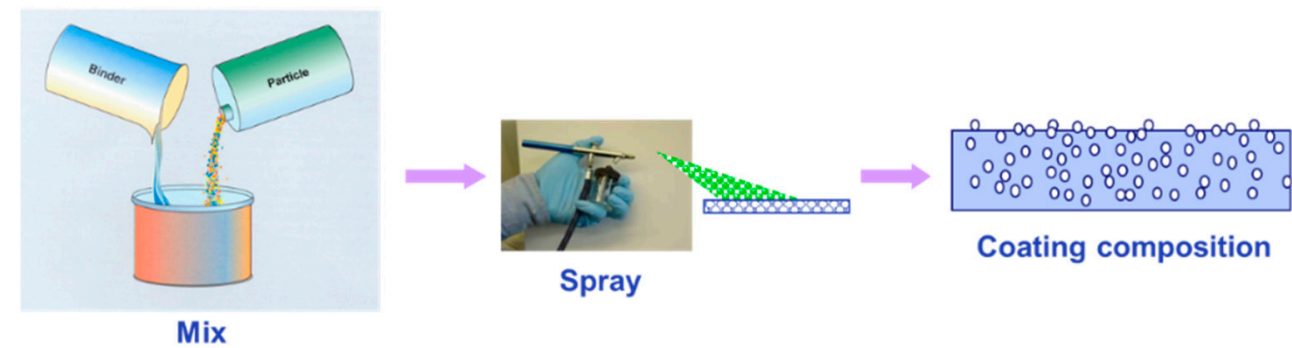

Figure 3. Schematic diagram of the preparation process of the superhydrophobic coating based on particles and binder. 


\subsection{Characterization}

The contact angle and roll-off angle of the coating were measured by Optical Contact Angle Meter OCA300 (Dataphysics, Filderstadt, Germany). The morphology of the coating surface and cross section were captured using scanning electron microscopy (SEM) (JSM-6460LV, JEOL, Tokyo, Japan). The ${ }^{29} \mathrm{Si}$ solid-state NMR spectra were obtained using Bruker Avance $400 \mathrm{MHz}$ NMR spectrometer (Billerica, MA, USA), cross polarization (CP) and magic-angle spinning (MAS).

The coating integrity was evaluated by a manually performed Q-tip abrasion test, and the superhydrophobicity of the areas after Q-tip abrasion was tested by water droplet roll-off behavior; the detailed rating criteria are described in our previous paper [24]. The higher the rating, the better the abrasion resistance and the superhydrophobicity.

\section{Results and Discussion}

\subsection{Surface Functionalization of Colloidal Silica}

The procedure for the functionalization of the colloidal silica is shown schematically in Figure 4. In order to maximize the functional degree of the colloidal silica, an excess of fluorine silane was added.

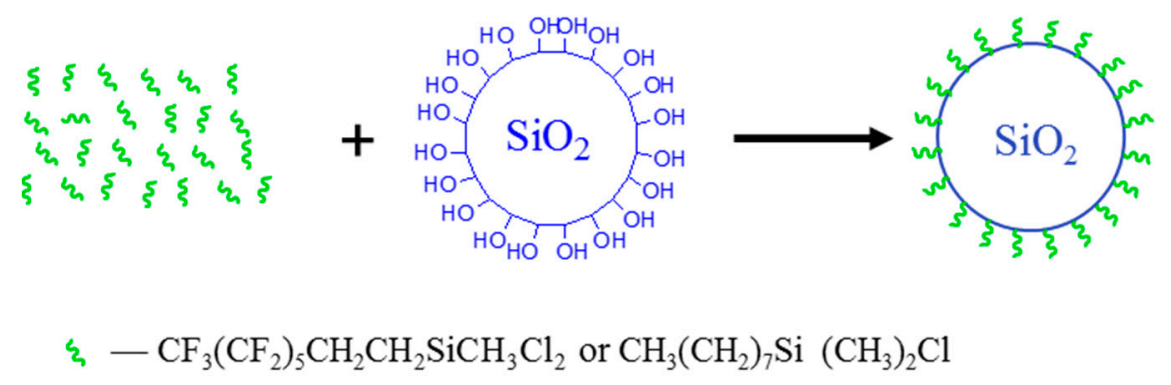

Figure 4. Schematic diagram of the functionalization of the colloidal particle.

To prove the functional fluorine group, which can be successfully attached to the colloidal silica with a particle size of $340 \mathrm{~nm}$, first, XPS elemental analysis was used to detect the F element on the modified colloidal silica surface. The XPS results of the colloidal silica and fluorine-functionalized colloidal silica are shown in Table 1 . The results clearly show that the percentage of fluorine element $(\mathrm{F})$ in the fluorine-modified silica particles $(28.66 \%)$ was significantly higher than that of the unmodified sample $(2.47 \%)$, thereby verifying that the fluorine functional groups were successfully attached to the surface of the silica particles.

Table 1. X-ray photoelectron spectroscopy (XPS) analysis of F-functionalized colloidal silica.

\begin{tabular}{ccccc}
\hline Sample & C (\%) & O (\%) & Si (\%) & F (\%) \\
\hline Colloidal silica & 19.28 & 59.64 & 18.60 & 2.47 \\
F-colloidal silica & 18.21 & 38.42 & 14.71 & 28.66 \\
\hline
\end{tabular}

In addition to determining the presence of the fluorine element on the surface of the silica particles, it is necessary to determine the chemical bonding, as opposed to the physical adsorption of the fluorine element on the silica surface. The condensation degree of the organosilane with the silanol on the surface of the silica particles can help to provide confirmation of the chemical bonding, as well as provide information on the extent of functionalization. ${ }^{29} \mathrm{Si}$ solid-state NMR is commonly used to determine the degree of condensation of silanol groups on the surface of silica [28,29]. An insight into organosilane-silanol condensation is substantiated by the presence of peaks in the ${ }^{29} \mathrm{Si}$ absorption associated with colloidal silica. The degree of condensation around the silicon atom could be represented by the ${ }^{29} \mathrm{Si}$ peaks, which are typically referred to as the ' $\mathrm{Q}$ ' peaks, including $\mathrm{Q} 1$, $\mathrm{Q} 2, \mathrm{Q} 3$, and Q4. A detailed description of Q peaks is described in our previous paper [24]. The ${ }^{29} \mathrm{Si}$ 
solid-state NMR spectrum of fluorine-modified colloidal silica and unmodified silica with a particle size of $340 \mathrm{~nm}$ is shown in Figure 5. However, there was almost no difference in the NMR spectra between the modified colloidal silica and the unmodified silica. This result could be attributed to two possible reasons: (1) no organosilane-silica condensation occurred, and the fluorine group was not chemically bonded to the colloidal silica, or (2) the degree of condensation of the silanol groups on the colloidal silica particles was too low to be detected. Usually the detection limit of ${ }^{29} \mathrm{Si}$ solid-state NMR is $1 \%$. According to the literature, the number of silanol groups on silica surfaces is about 3 per square $\mathrm{nm}$ [30]. If these silanols are all condensed with fluorine silane, the ratio of silicon atoms connected to fluorine functions to total silicon atoms can be calculated. The ratio is about $0.3 \%$ for the colloidal silica with a particle size of $340 \mathrm{~nm}$, which is lower than the detection limit of ${ }^{29} \mathrm{Si}$ solid-state NMR.

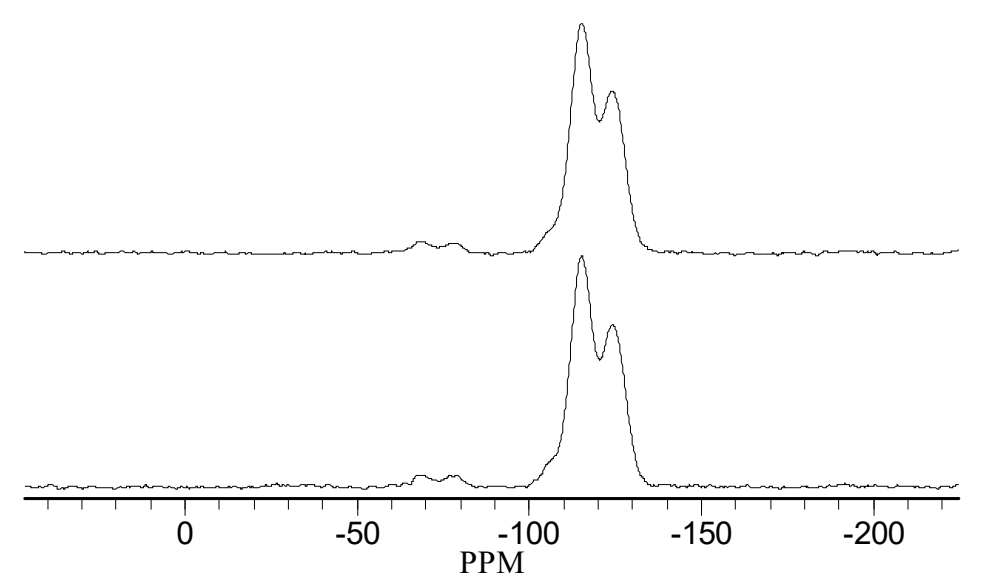

Figure $5 .{ }^{29} \mathrm{Si}$ solid-state NMR spectra of the fluorine-modified colloidal silica and unmodified silica (particle size: $340 \mathrm{~nm}$ ).

For the same weight of colloidal silica, silica with a smaller particle size will have much more surface area and many more silanol groups on the surface. Thus, the ratio of silicon atoms linked to the fluorine functional group to total silicon atoms will be much higher and can be detected. In order to confirm the chemical bonding between the colloidal silica and organosilane, a colloidal silica with a particle size of $12 \mathrm{~nm}$ was selected as a model system. Following the same functionalization procedure, a fluorine-functionalized silica with a particle size of $12 \mathrm{~nm}$ was prepared. According to the same calculation, the ratio is about $4.5 \%$ when all the silanols on the silica surface are assumed to react with the fluorine-silane, and this value is beyond the solid-NMR's limitation, making it easy to find the difference. Therefore, colloidal silica with a particle size of $12 \mathrm{~nm}$, functionalized by the same process, was used to verify the chemical bonding of the fluorine group on the surface of colloidal silica. The ${ }^{29} \mathrm{Si}$ solid-state NMR spectra of fluorine-modified colloidal silica and unmodified silica with a particle size of $12 \mathrm{~nm}$ are shown in Figure 6. After surface modification, the decrease of Q2 and the increase of $\mathrm{Q} 4$ relative to $\mathrm{Q} 3$ indicate that the amount of silanol groups was reduced, and the silanol groups on the surface of the silica particles had reacted with fluorine silane. The new peaks at about -20 and $-30 \mathrm{ppm}$ belong to silicon connected to the fluorine functional group. The results confirm the condensation of fluorine-silane and silanol groups and the chemical bonding of the fluorine group on the surface of the colloidal silica. For the octyl group-modified colloidal silica, the same functionalization process was followed, and the condensation of the octylsilane and silanol groups was the same as that of the fluorine-silane and silanol groups. 


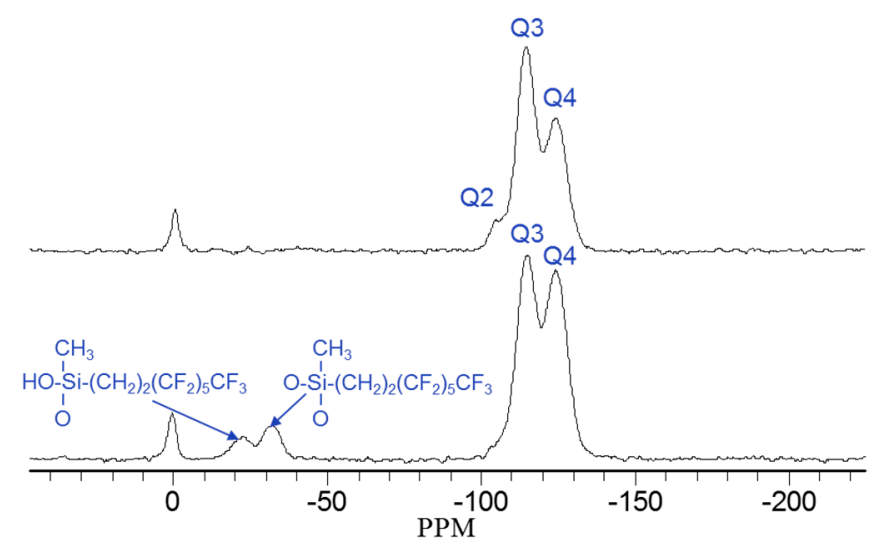

Figure $6 .{ }^{29} \mathrm{Si}$ solid-state NMR spectra of the fluorine-modified colloidal silica and unmodified silica (particle size: $12 \mathrm{~nm}$ ).

\subsection{Superhydrophobic Coating with Fluorine-Free Polymer}

The superhydrophobic coating, based on fluorine-functionalized colloidal silica and fluoropolymer, showed excellent mechanical properties in our previous study. However, a serious environmental concern is raised by fluorine materials, because they release highly toxic substances into the environment, which persist and accumulate in organisms. Thus, a fluorine-free superhydrophobic coating system is expected to be the most effective way to solve this issue. In our previous work, a superhydrophobic coating with good mechanical properties, which is based on fluorine-functionalized colloidal silica and fluorocopolymer, was successfully developed. Here, starting from this successful system, we planned to replace the fluorocopolymer with fluorine-free polymers, and thus, a system with fluorine-functionalized colloidal silica and fluorine-free polymers would be investigated to develop a coating with superhydrophobicity and good mechanical properties. If this system using fluorine-functionalized colloidal silica and fluorine-free polymers was successful, then we planned to replace the fluorine-functionalized colloidal silica with fluorine-free functionalized colloidal silica to investigate its superhydrophobicity and mechanical properties once again. To prepare the desired system without any fluorine materials, a system of fluorine-functionalized colloidal silica and fluorine-free polymers was investigated first.

Excepting fluorine polymer, silicone and olefin are the most promising materials with low surface energy and good hydrophobicity. Because cross-linkable silicone polymers are most efficient at providing better mechanical properties, a series of cross-linkable pure silicone resins and modified silicone resins were selected as polymer binders for the superhydrophobic coating. In our previous study, the optimized superhydrophobic coating formulation with good coating integrity was identified, based on $340 \mathrm{~nm}$ fluorine-functionalized particles and a fluorine polymer with a particle concentration of $60 \%$ volume. Thus, in this paper, fluorine-functionalized colloidal silica particles with a concentration volume of $60 \%$ were mixed with silicone resins, and the feasibility of a silicone polymer-based superhydrophobic coating was studied.

In this paper, cross-linkable pure silicone resins and modified silicone resins were the purchased from Momentive Advanced Materials. The types of pure silicone resins used were TSR116, TSR144, TSR145, and TSR1278, and the major differences among these silicone resins were a different ratio of methyl content $(\mathrm{Me} \%)$ and a different ratio of tri-functional groups $(\mathrm{T} \%)$. The properties of these pure silicone resins are shown in Figure 7. A silicone resin with more tri-functional groups has a higher cross-linking degree and is supposed to have better mechanical properties. Each pure silicone resin was mixed with fluorine-functionalized colloidal silica, and thus, a series of superhydrophobic coatings was prepared by following the process as described in Section 2. 


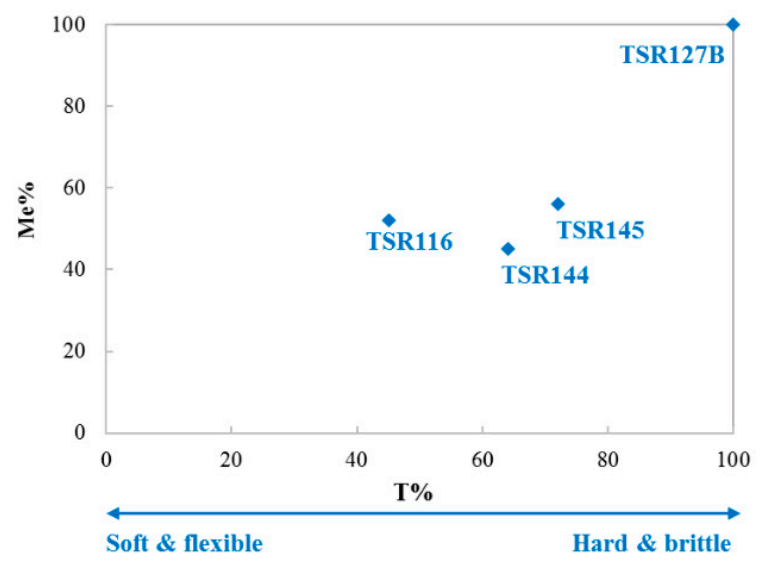

Figure 7. Properties of different pure silicone resins.

The properties of the superhydrophobic coatings are shown in Table 2. All of the coatings are superhydrophobic, but the coating integrity varies. The coatings using silicone resin with higher cross-linking degree show better coating integrity, but the coating integrity is still not sufficient when compared with the fluoropolymer-based coatings in our previous work. Thus, to obtain better coating integrity, modified silicone resins were used to prepare a superhydrophobic coating.

Table 2. Properties of the superhydrophobic coatings based on pure silicone resin and fluorine-functionalized colloidal silica.

\begin{tabular}{ccccc}
\hline Resin & Contact Angle $\left(^{\circ}\right)$ & Roll-Off Angle $\left(^{\circ}\right)$ & Q-Tip Scratch Rating & $\begin{array}{c}\text { Roll-off Rating } \\
\text { after Abrasion }\end{array}$ \\
\hline TSR116 & 151 & 5 & 1 & 1 \\
TSR144 & 158 & 2 & 2 & 3 \\
TSR145 & 154 & 2 & 2 & 2 \\
TSR127B & 152 & 1 & 2 & 2 \\
F-resin (control) & 155 & 1 & 3 & 3 \\
\hline
\end{tabular}

The types of modified silicone resins used were TSR194, TSR175, and TSR171. Different types correspond to different modified groups, of which TSR194 is an epoxy-modified silicone resin, TSR175 is a two-component urethane-modified silicone resin, and TSR171 is an acrylic-modified silicone resin. The modified silicone resin is mixed with fluorine-functionalized colloidal silica and a series of superhydrophobic coatings was prepared by following the same process as described in Section 2. The properties of the superhydrophobic coatings are shown in Table 3. The epoxy-modified silicone resin TSR194 shows good superhydrophobicity and good coating integrity, which is identical to the fluoropolymer-based superhydrophobic coating. This is attributed to the high crosslinking degree of the epoxy-modified silicone resin and the good mechanical properties of the epoxy polymer.

Table 3. Properties of the superhydrophobic coatings based on modified silicone resin and fluorine-functionalized colloidal silica.

\begin{tabular}{ccccc}
\hline Resin & Contact Angle $\left(^{\circ}\right)$ & Roll-Off Angle $\left(^{\circ}\right)$ & Q-Tip Scratch Rating & $\begin{array}{c}\text { Roll-Off Rating } \\
\text { After Abrasion }\end{array}$ \\
\hline TSR194 & 153 & 4 & 3 & 3 \\
TSR175 & 151 & 6 & 2 & 1 \\
TSR171 & 152 & 6 & 1 & 1 \\
F-resin (control) & 155 & 1 & 3 & 3 \\
\hline
\end{tabular}

\subsection{Fluorine-Free Superhydrophobic Coating}

The coating based on epoxy-modified silicone resin TSR194 and fluorine-functionalized colloidal silica shows good superhydrophobicity and a good coating integrity. In order to obtain the fluorine-free 
superhydrophobic coating, octyl chlorosilane was used to modify the colloidal silica with a particle size of $340 \mathrm{~nm}$, following the same surface modification process. The octyl group-modified silica particles and TSR194 resin were mixed, and the concentration of the silica particles was controlled to be $60 \%$ volume. The superhydrophobic coating was prepared by spraying and then by thermal curing. Typical SEM images of the coating surface and cross section are shown in Figure 8, which show that the coating surface is moderately rough. The specific properties of these coatings are shown in Table 4 . The coating based on TSR194 and the octyl-modified colloidal silica shows good superhydrophobicity and good mechanical properties. In order to investigate and test the coating robustness and water resilience, the prepared coating was immersed in water for 7 days. The superhydrophobicity and coating integrity were measured. There was no change in the coating performance after being immersed in water for 7 days. When the superhydrophobic coating was applied to the stainless-steel plate by the same process, the same performance was obtained. Therefore, the results prove the feasibility of a fluorine-free superhydrophobic coating with good coating integrity and present an effective solution to the environmental problems caused by fluorine chemicals by using fluorine-free raw materials.

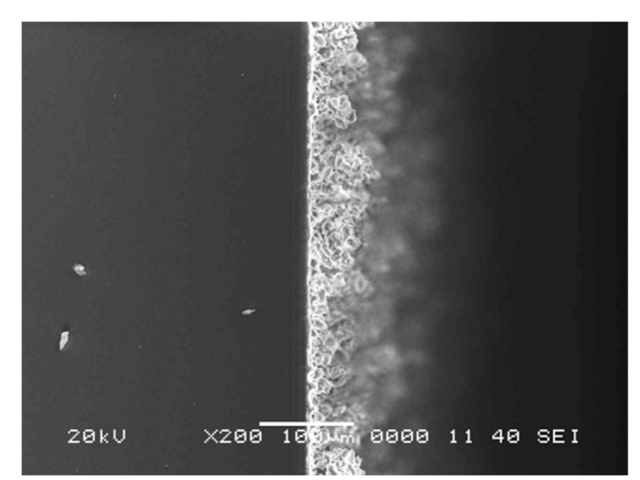

(a)

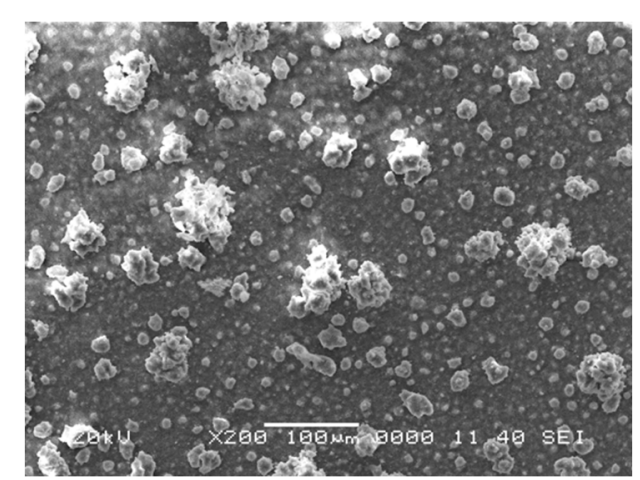

(b)

Figure 8. Surface and cross-section SEM images of a coating based on TSR194 and octyl-modified colloidal silica. (a) cross-section SEM image; (b) surface SEM image.

Table 4. Properties of the superhydrophobic coating based on epoxy-modified silicone resin and octyl-functionalized colloidal silica.

\begin{tabular}{ccccc}
\hline Resin & Contact Angle $\left(^{\circ}\right)$ & Roll-Off Angle ( ${ }^{\circ}$ ) & Q-Tip Scratch Rating & $\begin{array}{c}\text { Roll-Off Rating } \\
\text { After Abrasion }\end{array}$ \\
\hline TSR144+ & 153 & 4 & 3 & 3 \\
Octyl-SiO & & 1 & 3 & 3 \\
F-resin (control) & 155 & & 3 \\
\hline
\end{tabular}

\section{Conclusions}

Silicone materials, due to their good hydrophobicity, are the most promising candidates to replace fluorine materials, which have recently raised serious environmental concern. Based on our previous work, in this paper, fluorine components were replaced by fluorine-free materials step by step to develop a fluorine-free superhydrophobic coating. First, the fluorocopolymer was replaced by silicone materials. Then, octyl-modified colloidal silica was prepared to replace the fluorine-modified colloidal silica. Thus, a fluorine-free superhydrophobic coating based on epoxy-modified silicone and octyl-modified colloidal silica was achieved, and the developed coating shows both good superhydrophobicity and good coating integrity. This result demonstrates the feasibility of a fluorine-free superhydrophobic coating with good coating integrity and provides an effective solution to the environmental problems caused by fluorine chemicals.

Author Contributions: Conceptualization, H.L.; Methodology, H.L.; Investigation, H.L., J.X., M.X. and L.Z.; Resources, J.X. and Q.Z.; Data Curation, H.L. and M.X.; Writing-Original Draft Preparation, H.L.; Writing-Review and Editing, Q.Z. 
Funding: This research received no external funding.

Acknowledgments: Many thanks to the GE Global Research Shanghai team members and the Niskayuna team members for their generous help with the experiments and sharing of knowledge.

Conflicts of Interest: The authors declare no conflicts of interest or other financial conflicts.

\section{References}

1. Blossey, R. Self-cleaning surfaces-Virtual realities. Nat. Mater. 2003, 2, 301-306. [CrossRef] [PubMed]

2. Otten, A.; Herminghaus, S. How plants keep dry: A physicist's point of view. Langmuir 2004, 20, $2405-2408$. [CrossRef] [PubMed]

3. Watson, G.S.; Watson, J.A. Natural nano-structures on insects-possible functions of ordered arrays characterized by atomic force microscopy. Appl. Surf. Sci. 2004, 235, 139-144. [CrossRef]

4. Neinhuis, C.; Barthlott, W. Characterization and distribution of water-repellent, self-cleaning plant surfaces. Ann. Bot. 1997, 79, 667-677. [CrossRef]

5. Barthlott, W.; Neinhuis, C. Purity of the sacred lotus, or escape from contamination in biological surfaces. Planta 1997, 202, 1-8. [CrossRef]

6. Yamamoto, M.; Nishikawa, N.; Mayama, H.; Nonomura, Y.; Yokojima, S.; Nakamura, S.; Uchida, K. Theoretical explanation of the lotus effect: Superhydrophobic property changes by removal of nanostructures from the surface of a lotus leaf. Langmuir 2015, 31, 7355-7363. [CrossRef] [PubMed]

7. Zulfiqar, U.; Awais, M.; Hussain, S.Z.; Hussain, I.; Husain, S.W.; Subhani, T. Durable and self-healing super-hydrophobic surfaces for building materials. Mater. Lett. 2017, 192, 56-59. [CrossRef]

8. Junaidi, M.U.M.; Haji Azaman, S.A.; Ahmad, N.N.R.; Leo, C.P.; Lim, G.W.; Chan, D.J.C.; Yee, H.M. Super-hydrophobic coating of silica with photoluminescence properties synthesized from rice husk ash. Prog. Org. Coat. 2017, 111, 29-37. [CrossRef]

9. Gao, S.; Dong, X.; Huang, J.; Li, S.; Li, Y.; Chen, Z.; Lai, Y. Rational construction of highly transparent super-hydrophobic coatings based on a non-particle, fluorine-free and water-rich system for versatile oil-water separation. Chem. Eng. J. 2018, 333, 621-629. [CrossRef]

10. Zeng, X.; Qian, L.; Yuan, X.; Zhou, C.; Li, Z.; Cheng, J.; Xu, S.; Wang, S.; Pi, P.; Wen, X. Inspired by stenocara beetles: From water collection to highefficiency water-in-oil emulsion separation. ACS Nano 2017, 11, 760-769. [CrossRef]

11. Liu, Y.; Song, D.; Choi, C.H. Anti- and de-icing behaviors of super-hydrophobic fabrics. Coatings 2018, 8, 198. [CrossRef]

12. Jiang, G.; Chen, L.; Zhang, S.; Huang, H. Super-hydrophobic SiC/CNTs coatings with photothermal deicing and passive anti-icing properties. ACS Appl. Mater. Interfaces 2018, 10, 36505-36511. [CrossRef] [PubMed]

13. Lin, Y.; Chen, H.; Wang, G.; Liu, A. Recent progress in preparation and anti-icing applications of super-hydrophobic coatings. Coatings 2018, 8, 208. [CrossRef]

14. Zheng, S.; Li, C.; Fu, Q.; Hu, W.; Xiang, T.; Wang, Q.; Du, M.; Liu, X.; Chen, Z. Development of stable super-hydrophobic coatings on aluminum surface for corrosion-resistant, self-cleaning, and anti-icing applications. Mater. Des. 2016, 93, 261-270. [CrossRef]

15. Zhao, Q.; Tang, T.; Wang, F. Fabrication of super-hydrophobic AA5052 aluminum alloy surface with improved corrosion resistance and self cleaning property. Coatings 2018, 8, 390. [CrossRef]

16. Sebastian, D.; Yao, C.; Lian, I. Mechanical durability of engineered super-hydrophobic surfaces for anti-corrosion. Coatings 2018, 8, 162. [CrossRef]

17. Pan, G.; Xiao, X.; Yu, N.; Ye, Z. Fabrication of super-hydrophobic coatings on cotton fabric using ultrasound-assisted in-situ growth method. Prog. Org. Coat. 2018, 125, 463-471. [CrossRef]

18. Huang, S.; Liu, G.; Zhang, K.; Hu, H.; Wang, J.; Miao, L.; Tabrizizadeh, T. Water-based polyurethane formulations for robust super-hydrophobic fabrics. Chem. Eng. J. 2019, 360, 445-451. [CrossRef]

19. Wang, N.; Tang, L.; Cai, Y.; Tong, W.; Xiong, D. Scalable super-hydrophobic coating with controllable wettability and investigations of its drag reduction. Coll. Surf. A Physicochem. Eng. Asp. 2018, 555, 290-295. [CrossRef]

20. Tuo, Y.; Chen, W.; Zhang, H.; Li, P.; Liu, X. One-step hydrothermal method to fabricate drag reduction super-hydrophobic surface on aluminum foil. Appl. Surf. Sci. 2018, 446, 230-235. [CrossRef] 
21. Dimitrakellis, P.; Gogolides, E. Atmospheric plasma etching of polymers: A palette of applications in cleaning/ashing, pattern formation, nanotexturing and super-hydrophobic surface fabrication. Microelectron. Eng. 2018, 194, 109-115. [CrossRef]

22. Ma, M.; Mao, Y.; Gupta, M.; Gleason, K.K.; Rutledge, G.C. Super-hydrophobic fabrics produced by electrospinning and chemical vapor deposition. Macromolecules 2005, 38, 9742-9748. [CrossRef]

23. Nakajima, A.; Abe, K.; Hashimoto, K.; Watanabe, T. Preparation of hard super-hydrophobic films with visible light transmission. Thin Solid Films 2000, 376, 140-143. [CrossRef]

24. Lei, H.; Xiao, J.; Zheng, L.; Xiong, M.; Zhu, Y.; Qian, J.; Zhuang, Q.; Han, Z. Super-hydrophobic coatings based on colloid silica and fluorocopolymer. Polymer 2016, 86, 22-31. [CrossRef]

25. Renner, R. Piecing together the perfluorinated puzzle. Anal. Chem. 2005, 77, 15A-16A. [CrossRef]

26. Renner, R. The long and the short of perfluorinated replacements. Environ. Sci. Technol. 2006, 40, 12-13. [CrossRef] [PubMed]

27. Vierke, L.; Staude, C.; Biegel-Engler, A.; Drost, W.; Schulte, C. Perfluorooctanoic acid (PFOA)-main concerns and regulatory developments in Europe from an environmental point of view. Environ. Sci. Europe 2012, 24, 16. [CrossRef]

28. Legrand, A.P.; Hommel, H.; Taibi, H.; Miquel, J.L.; Tougne, P. Contribution of solid state NMR spectroscopy to the characterization of materials. Coll. Surf. 1990, 45, 391-411. [CrossRef]

29. Leonardelli, S.; Facchini, L.; Fretigny, C.; Tougne, P.; Legrand, A.P. Silicon-29 NMR study of silica. J. Am. Chem. Soc. 1992, 114, 6412-6418. [CrossRef]

30. Duvault, Y.; Gagnaire, A.; Gardies, F.; Jaffrezic-Renault, N.; Martelet, C.; Morel, D.; Serpinet, J.; Duvault, J.L. Physicochemical characterization of covalently bonded alkyl monolayers on silica surfaces. Thin Solid Films 1990, 185, 169-179. [CrossRef]

(C) 2019 by the authors. Licensee MDPI, Basel, Switzerland. This article is an open access article distributed under the terms and conditions of the Creative Commons Attribution (CC BY) license (http:/ / creativecommons.org/licenses/by/4.0/). 